\title{
MORSE THEORY AND GEODESICS IN THE SPACE OF KÄHLER METRICS
}

\author{
TAMÁS DARVAS \\ (Communicated by Lei $\mathrm{Ni}$ ) \\ To my parents
}

\begin{abstract}
Given a compact Kähler manifold $\left(X, \omega_{0}\right)$ let $\mathcal{H}_{0}$ be the set of Kähler forms cohomologous to $\omega_{0}$. As observed by Mabuchi, this space has the structure of an infinite dimensional Riemannian manifold if one identifies it with a totally geodesic subspace of $\mathcal{H}$, the set of Kähler potentials of $\omega_{0}$. Following Donaldson's research program, existence and regularity of geodesics in this space is of fundamental interest. In this paper, supposing enough regularity of a geodesic $u:[0,1] \rightarrow \mathcal{H}$, connecting $u_{0} \in \mathcal{H}$ with $u_{1} \in \mathcal{H}$, we establish a Morse theoretic result relating the critical points of $u_{1}-u_{0}$ to the critical points of $\dot{u}_{0}=d u /\left.d t\right|_{t=0}$. As an application of this result, we prove that on all Kähler manifolds, connecting Kähler potentials with smooth geodesics is not possible in general. In particular, in the case $X \neq \mathbb{C} P^{1}$, we will also prove that the set of pairs of potentials that cannot be connected with smooth geodesics has nonempty interior. This is an improvement upon the findings of Lempert and Vivas and of the author and Lempert.
\end{abstract}

\section{INTRODUCTION}

Let $\left(X^{n}, \omega_{0}\right)$ be a connected compact Kähler manifold. The space of smooth Kähler potentials of $\omega_{0}$ is the set

$$
\mathcal{H}:=\left\{v \in C^{\infty}(X): \omega_{0}+i \partial \bar{\partial} v>0\right\} .
$$

It is clear that $\mathcal{H}$ is a Fréchet manifold, as it is an open subset of $C^{\infty}(X)$. From this, it also follows that for each $v \in \mathcal{H}$ we can identify $T_{v} \mathcal{H}$ with $C^{\infty}(X)$. Following Mabuchi, we define a Riemannian metric on $T_{v} \mathcal{H}$ :

$$
\langle\xi, \eta\rangle:=\int_{X} \xi \eta\left(\omega_{0}+i \partial \bar{\partial} v\right)^{n}, \quad \xi, \eta \in T_{v} \mathcal{H} .
$$

If $\phi:[0,1] \rightarrow \mathcal{H}$ is a smooth curve, one can think of $\phi$ as an element of $C^{\infty}([0,1] \times$ $X)$. Also, suppose that $\psi$ is a tangent vector field along $\phi$, which again can be treated as an element of $C^{\infty}([0,1] \times X)$. The Levi-Civita connection of the metric introduced above is as follows:

$$
\nabla_{\dot{\phi}} \psi:=\dot{\psi}-\frac{1}{2}\langle\nabla \psi, \nabla \dot{\phi}\rangle
$$

Received by the editors September 4, 2012.

2010 Mathematics Subject Classification. Primary 32Q15, 32W20.

This research was supported by NSF grant DMS 1162070 and the Purdue Research Foundation. 
Hence a smooth curve $g:[0,1] \rightarrow \mathcal{H}$ is a geodesic if we have

$$
0=\nabla_{\dot{g}} \dot{g}=\ddot{g}-\frac{1}{2}\langle\nabla \dot{g}, \nabla \dot{g}\rangle \text {. }
$$

Let us now relate the above discussion to the space of Kähler metrics over $X$. Hodge theory implies that the correspondence $u \longrightarrow \omega_{0}+i \partial \bar{\partial} u$ gives an onto map from $\mathcal{H}$ to $\mathcal{H}_{0}$. As found by Mabuchi, $\mathcal{H}_{0}$ can be identified with a totally geodesic subspace of $\mathcal{H}$. This correspondence will automatically put a Riemannain structure on $\mathcal{H}_{0}$. Moreover, one can prove that with this structure, $\mathcal{H}$ is isometric to the Riemannian product $\mathcal{H}_{0} \times \mathbb{R}$. Hence to study the question of existence of geodesics in $\mathcal{H}_{0}$ one can study the same question in $\mathcal{H}$.

As discovered by Semmes $[\underline{\mathrm{S}}$, the geodesic equation can be rewritten as a complex Monge-Ampère equation. Let $S=\{s \in \mathbb{C}: 0<\operatorname{Im} s<1\}$, and let $\omega$ be the pullback of the Kähler form $\omega_{0}$ to $\bar{S} \times X$ by the projection $\pi_{X}: S \times X \rightarrow X$. If $\phi:[0,1] \rightarrow \mathcal{H}$ is a smooth curve, let $u \in C^{\infty}(\bar{S} \times X)$ be defined by $u(s, x):=\phi(\operatorname{Im} s, x)$. Semmes observed that $\phi$ is a geodesic if and only if the following equation is satisfied for $u$ :

$$
(\omega+i \partial \bar{\partial} u)^{n+1}=0 .
$$

Since the form $\omega+\partial \bar{\partial} u$ is nondegenerate on each $X$-fiber, the above equation is the same as writing $(\omega+i \partial \bar{\partial} u)^{n+1}=0$ but $(\omega+i \partial \bar{\partial} u)^{n} \neq 0$. Summing up, we can say that if there exists a geodesic connecting $u_{0} \in \mathcal{H}$ with $u_{1} \in \mathcal{H}$, then there exists a smooth function $u \in C^{\infty}(\bar{S} \times X)$ that satisfies the following conditions:

$$
\begin{aligned}
& (\omega+i \partial \bar{\partial} u)^{n+1}=0, \\
& (\omega+i \partial \bar{\partial} u)^{n} \neq 0, \\
& u(r+i t, x)=u(i t, x) \forall x \in X, t \in[0,1], r \in \mathbb{R}, \\
& u(0, x)=u_{0}(x), u(i, x)=u_{1}(x) \forall x \in X .
\end{aligned}
$$

Solutions to the above problem have been intensely studied. Uniqueness has already been found by Donaldson [D1]. Chen [C] proved that one always has a $\omega$-plurisubharmonic solution to (1.1) in the sense of Bedford and Taylor BT] for which the current $\partial \bar{\partial} u$ is represented by a bounded form. Of course in this setting the condition $(\omega+i \partial \bar{\partial} u)^{n} \neq 0$ does not make any sense, and hence it is omitted from the problem.

In [LV] the authors proved that on certain Kähler manifolds admitting special symmetries there exist boundary data for which (1.1) has no $C^{3}$ solutions. This provided the first example showing that, using smooth geodesics, connecting points in $\mathcal{H}$ is not possible in general. In $\mathrm{DL}$, under similar circumstances, it was shown that the regularity result of Chen we just mentioned is sharp.

In this paper we only investigate $C^{3}$ solutions of problem (1.1). More specifically, with the above conventions in mind, we prove the following result:

Theorem 1.1. Suppose $u$ is a $C^{3}$ solution of (1.1). Then all the critical points of $\dot{u}_{0}:=d u(i t, \cdot) /\left.d t\right|_{t=0} \in C^{2}(X)$ are among the critical points of $u_{1}-u_{0}$. Moreover, if $u_{1}-u_{0}$ is a Morse function, then $\dot{u}_{0}$ is a Morse function as well.

The above result establishes a connection between the boundary data and initial tangent vector of $C^{3}$ solutions of the complex Monge-Ampère equation. This 
theorem is proved by a careful analysis of the first order variation of the MongeAmpère foliation of $u$ at each critical point of the initial tangent vector $\dot{u}_{0}$. A similar method has been applied with success in [LV] as well. As an application we prove the following theorem:

Theorem 1.2. For each connected compact Kähler manifold $\left(X, \omega_{0}\right)$ one can find a pair of potentials $u_{0}, u_{1} \in \mathcal{H}$ that cannot be connected by a smooth geodesic. If $X \neq \mathbb{C} P^{1}$, then the set of such pairs of potentials has nonempty interior in $C^{\infty}(X) \times C^{\infty}(X)$.

This result is an improvement upon the similar nonexistence result of [LV]. The examples constructed there only work on manifolds admitting holomorphic isometries $A: X \rightarrow X$ having an isolated fixed point and satisfying the identity $A^{2}=I d$.

In Kähler geometry the special role of symmetries, especially that of oneparameter group actions, has a long history. The first result of this kind was that of Matushima [M], who proved that if the Lie algebra of holomorphic vector fields on a complex manifold is not reductive, then the manifold admits no Kähler-Einstein metrics. By contrast, in the absence of holomorphic vector fields, the search for canonical Kähler metrics becomes more promising. Calabi Ca observed that in this case, all extremal metrics will have constant scalar curvature. The Futaki invariant, that serves as an obstruction to finding constant scalar curvature metrics, vanishes in this situation as well $[\mathrm{F}$.

With the above picture and the examples of [LV] in mind, one might perhaps think that constructing pairs of Kähler potentials that cannot be connected by a smooth geodesic requires the presence of holomorphic symmetries. The interest of Theorem 1.2 is showing that this is not the case.

It would be interesting to see if one can prove the last statement of the above result for $\mathbb{C} P^{1}$ as well. We summarize the proof of Theorem 1.2 here. In the case $X=\mathbb{C} P^{1}$ every Kähler metric $\omega_{0}$ is in the Kähler class of some multiple of the Fubini-Study metric $\omega_{F S}$. However, each metric $c \omega_{F S}$ satisfies the symmetry conditions of Theorem 1.1 in [LV], and so this theorem provides the $u_{0}, u_{1} \in \mathcal{H}$ that we seek. Hence, to prove the result, we only have to consider the case $X \neq$ $\mathbb{C} P^{1}$. The rest of the argument will be a consequence of the following more precise statement:

Theorem 1.3. Suppose $X \neq \mathbb{C} P^{1}$. For each connected compact Kähler manifold $\left(X, \omega_{0}\right)$ one can find boundary data $u_{0}, u_{1} \in \mathcal{H}$ for which problem (1.1) has no $C^{3}$ solutions. In particular, the set of such pairs has nonempty interior in $\mathcal{H}$.

\section{The Monge-Ampère Foliation}

In this section we summarize known facts about the Monge-Ampère foliation, first discusessed in $[\overline{B K}$. For a more complete picture, the reader is referred to [D2] or Section 2.1 in RZ.

The reason $C^{3}$ solutions of the complex Monge-Ampère equation are special is that this is the minimal amount of regularity that is needed for the existence of the Monge-Ampère foliation. This is defined as follows. Let $u \in C^{\infty}(\bar{S} \times X)$ be a solution of (1.1). This means that for each $(s, x)=(r+i t, x) \in \bar{S} \times X$, $\left.\operatorname{Ker}(\omega+i \partial \bar{\partial} u)\right|_{(s, x)}$ is a one dimensional complex subspace of $T_{(s, x)}(\bar{S} \times X)$, called the Monge-Ampère distribution. Since the form $\omega+i \partial \bar{\partial} u$ is closed, this complex 
subbundle of $T(\bar{S} \times X)$ is integrable, and hence gives rise to a foliation in which each leaf is a one dimensional complex submanifold of $\bar{S} \times X$.

We will denote by $\mathcal{F}_{x}$ the leaf of this foliation passing through the point $(0, x)$. Also, $u_{t}(\cdot)$ and $\dot{u}_{t}(\cdot)$ will stand for $u(i t, \cdot)$ and $d u(i t, \cdot) / d t$ for $t \in[0,1]$. We should note that our formulation of (1.1) allows solutions $u$ for which some of the forms $\omega_{0}+i \partial_{X} \bar{\partial}_{X} u_{t}$ are degenerate on $X$. Because of this, in general, the leaves $\mathcal{F}_{x}$ cannot be assumed to be diffeomorphic to the strip $\bar{S}$. This will only be a minor inconvenience, as we will see in a moment.

As observed by Semmes $\left[\mathbf{S}\right.$, the vector field $\partial / \partial r+J \nabla_{g_{0}} \dot{u}_{0} / 2$ generates $\operatorname{Ker}(\omega+$ $i \partial \bar{\partial} u)\left.\right|_{(r, x)}$, where $g_{0}$ is the Riemannian metric corresponding to the Kähler metric $\omega_{0}+i \partial_{X} \bar{\partial}_{X} u_{0}$ and $x \in X, r \in \mathbb{R}$. Since $J \nabla_{g_{0}} \dot{u}_{0} / 2$ is independent of $r$, this has a very interesting consequence. If $r \rightarrow f_{x}(r), r \in \mathbb{R}$, is the trajectory of the vector field $J \nabla_{g_{0}} \dot{u}_{0} / 2$ with initial data $f_{x}(0)=x$, then the leaf $\mathcal{F}_{x}$ contains the image of the arc $r \rightarrow \Gamma_{x}(r)=\left(r, f_{x}(r)\right)$. Hence, with a slight abuse of precision, one should think of each leaf $\mathcal{F}_{x}$ as the unique analytic extension of the curve $\Gamma_{x}$. This fact will be used in the next section.

\section{Proof of Theorem 1.1}

By replacing $\omega_{0}$ with $\omega_{0}+i \partial \bar{\partial} u_{0}$ and $u(s, x)$ with $u(s, x)-u_{0}(x)$, we can assume that $u_{0}=0$. We prove Theorem 1.1 in two steps.

Lemma 3.1. If $x_{0}$ is a critical point of $\dot{u}_{0}$, then it is a critical point of $\dot{u}_{t}$ for all $t \in[0,1]$ and the leaf of the Monge-Ampère foliation through $x_{0}$ is $\bar{S} \times\left\{x_{0}\right\}$. In particular, $t \rightarrow u_{t}\left(x_{0}\right)$ is linear.

Proof. Denote $\omega_{t}=\omega_{0}+i \partial_{X} \bar{\partial}_{X} u_{t}$. We saw in the preceding section that the vector $\partial / \partial r+J \nabla_{g_{0}} \dot{u}_{0} / 2$ generates the Monge-Ampère distribution at any point $(r, x) \in \mathbb{R} \times X$.

Since $x_{0}$ is a critical point of $\dot{u}_{0}$, the vector field $J \nabla_{g_{0}} \dot{u}_{0} / 2$ vanishes at $x_{0}$, hence its trajectory $r \rightarrow f_{x_{0}}(r)$ is $x_{0}$. Therefore, following the reasoning of the preceding section, we obtain that $\mathcal{F}_{x_{0}}=\bar{S} \times\left\{x_{0}\right\}$. Hence, $\partial / \partial r$ generates $\operatorname{Ker}(\omega+$ $i \partial \bar{\partial} u)\left.\right|_{\left(s, x_{0}\right)}$ for any $s \in \bar{S}$. In any holomorphic chart of $X$ around $x_{0}$, this implies that $u_{\bar{s} z_{j}}\left(s, x_{0}\right)=u_{s \bar{z}_{j}}\left(s, x_{0}\right)=0$ and $u_{s \bar{s}}\left(s, x_{0}\right)=0$ for all $s \in \bar{S}$ and for all $j=1, \ldots, n$. As $u$ is translation invariant, $\dot{u}_{t}=2 i \partial u / \partial s, \ddot{u}_{t}=4 \partial^{2} u / \partial s \bar{\partial} s$ and we obtain the desired result.

From this lemma, we obtain that if $x_{0}$ is a critical point of $\dot{u}_{0}$, then it is a critical point of $u_{1}$ as well, since in any holomorphic chart around $x_{0}$ we have $u_{1 z_{j}}\left(x_{0}\right)=\int_{0}^{1} \dot{u}_{t z_{j}}\left(x_{0}\right) d t=0$ and $u_{1 \bar{z}_{j}}\left(x_{0}\right)=\int_{0}^{1} \dot{u}_{t \bar{z}_{j}}\left(x_{0}\right) d t=0$ for all $j=1, \ldots, n$. All that is left is to prove the nondegeneracy in Theorem 1.1. We will do this in the following lemma.

Lemma 3.2. If $x_{0}$ is a degenerate critical point of $\dot{u}_{0}$, then it is a degenerate critical point of $u_{1}$ as well. Moreover, for the Hessians of $\dot{u}_{0}$ and $u_{1}$ at $x_{0}$, we have the inclusion Ker $H \dot{u}_{0}\left(x_{0}\right) \subseteq$ Ker $H u_{1}\left(x_{0}\right)$.

Proof. Most of the computations to follow are taken over from [LV, Section 2]. We extend the Hessian forms of both $u_{1}$ and $\dot{u}_{0}$ to complex bilinear forms. Then we 
have the following representation of these forms at any point $x \in X$ in any complex coordinate:

$$
H u_{1}(x)=\left[\begin{array}{cc}
u_{1 z z}(x) & u_{1 z \bar{z}}(x) \\
u_{1 \bar{z} z}(x) & u_{1 \overline{z z}}(x)
\end{array}\right], H \dot{u}_{0}(x)=\left[\begin{array}{cc}
\dot{u_{0 z z}}(x) & \dot{u_{0 z \bar{z}}}(x) \\
\dot{u_{0 \bar{z} z}}(x) & \dot{u_{0 \bar{z}}}(x)
\end{array}\right],
$$

where $u_{1 z z}(x)=\left\{u_{1 z_{j} z_{k}}(x)\right\}_{j, k=1, n}$, and similarly for all the other terms.

We fix local coordinates $z_{j}, j=1, \ldots, n$, on a neighborhood $V \subset X$ of $x_{0}$ such that $\left.\omega_{0}\right|_{x_{0}}=\left.i d z_{j} \wedge d \bar{z}_{j}\right|_{x_{0}}$, the local coordinates map $V$ on a convex set in $\mathbb{C}^{n}$ and there exist a potential $w_{0}$ on $V$ such that $\omega_{0}=i \partial \bar{\partial} w_{0}$ on $V$. We can clearly suppose that $\left.w_{0 z_{j} z_{k}}\right|_{x_{0}}=0$. We denote by $w$ the function $\pi_{X}^{*} w_{0}$. We identify $V$ with its image in $\mathbb{C}^{n}$ and $x_{0}$ with $0 \in \mathbb{C}^{n}$. Then $\bar{S} \times V$ is identified with a subset of $\bar{S} \times \mathbb{C}^{n}$.

From Lemma 3.1 it follows that $f_{x_{0}}=f_{0} \equiv 0$ and the leaf of the Monge Ampère foliation passing through $\left(0, x_{0}\right)$ is $\bar{S} \times\left\{x_{0}\right\}$. As discussed in Section 2 of [LV], this implies that for any $d>0$ and for small enough $x$, it makes sense to holomorphically extend the trajectories $r \rightarrow f_{x}(r)$ from the segment $[-d, d] \subset \mathbb{R}$ to the compact set $\{z \in \bar{S} \mid-d \leq \operatorname{Re} z \leq d\}$. Because of our regularity assumptions, we have that the leaves of the Monge-Ampère foliation change differentiably. Hence for any $a \in \mathbb{C}^{n}$ one can define the functions $\varphi_{j}:=d f_{(t a)} /\left.d t\right|_{t=0}$, continuous on $\bar{S}$ with values in $\mathbb{C}$, that are holomorphic on $S$ for $j=1, \ldots, n$. It follows from basic properties of the Monge-Ampère foliation that whenever the maps below are defined, they are holomorphic:

$$
s \rightarrow w_{z_{j}}\left(s, f_{t a}(s)\right)+u_{z_{j}}\left(s, f_{t a}(s)\right), \quad j=1, \ldots, n .
$$

Differentiating the above maps with respect to $t$ we obtain that the functions

$$
s \rightarrow \sum_{k=1}^{n}\left(\left\{w_{z_{j} z_{k}}(s, 0)+u_{z_{j} z_{k}}(s, 0)\right\} \varphi_{k}(s)+\left\{w_{z_{j} \bar{z}_{k}}(s, 0)+u_{z_{j} \bar{z}_{k}}(s, 0)\right\} \overline{\varphi_{k}(s)}\right),
$$

$j=1, \ldots, n, s \in S$, are holomorphic as well. From our choice of $w_{0}$ it follows that $w_{z z}(s, 0) \equiv 0$ and $w_{z \bar{z}}(s, 0) \equiv I$. After differentiating the above maps by $\partial / \partial \bar{s}$ as well, we find that

$$
\sum_{k=1}^{n}\left(u_{z_{j} z_{k} \bar{s}}(s, 0) \varphi_{k}(s)+u_{z_{j} \bar{z}_{k} \bar{s}}(s, 0) \overline{\varphi_{k}(s)}+\left\{\delta_{j k}+u_{z_{j} \bar{z}_{k}}(s, 0)\right\} \overline{\varphi_{k}^{\prime}(s)}\right)=0,
$$

$j=1, \ldots, n$. Summing up, with the notation introduced at the beginning, we obtain that the function $\psi: S \rightarrow \mathbb{C}^{n}$ defined by

$$
\psi(s):=u_{z z}(s, 0) \varphi(s)+\left\{I+u_{z \bar{z}}(s, 0)\right\} \overline{\varphi(s)}
$$

is holomorphic and

$$
\left\{I+u_{\bar{z} z}(s, 0)\right\} \varphi^{\prime}(s)=-u_{\bar{z} z s}(s, 0) \varphi(s)-u_{\overline{z z} s}(s, 0) \overline{\varphi(s)} .
$$

With the notation $P=I+u_{z \bar{z}}(i, 0)=I+u_{1 z \bar{z}}(0), Q=u_{z z}(i, 0)=u_{1 z z}(0)$, $A=-u_{\bar{z} z s}(0,0)$ and $B=-u_{\bar{z} z s}(0,0)$, we find that

$$
\psi(z)= \begin{cases}\overline{\varphi(s)} & \text { if } \operatorname{Im} s=0 \\ P \overline{\varphi(s)}+Q \varphi(s) & \text { if } \operatorname{Im} s=1\end{cases}
$$

Restricting (3.1) to real $s$ we have

$$
\varphi^{\prime}(s)=A \varphi(s)+B \overline{\varphi(s)}, \quad s \in \mathbb{R} .
$$


We also observe that $\varphi(0)=a$. Hence, to find the values of $\varphi$, we need to solve the following initial value problem on the real line:

$$
\left[\begin{array}{c}
\varphi^{\prime}(s) \\
\bar{\varphi}^{\prime}(s)
\end{array}\right]=\left[\begin{array}{cc}
A & B \\
\bar{B} & \bar{A}
\end{array}\right]\left[\begin{array}{c}
\varphi(s) \\
\bar{\varphi}(s)
\end{array}\right],\left[\begin{array}{c}
\varphi(0) \\
\bar{\varphi}(0)
\end{array}\right]=\left[\begin{array}{c}
a \\
\bar{a}
\end{array}\right] .
$$

Since $A=i i_{0 \bar{z} z}(0) / 2, B=i i_{0 \overline{z z}}(0) / 2$ and $H \dot{u}(0)$ is assumed to be degenerate, we obtain that the matrix of the above linear initial value problem is degenerate too. This means that there exists a nonzero $\alpha \in \mathbb{C}^{n}$ such that

$$
\left[\begin{array}{c}
A \alpha+B \bar{\alpha} \\
\bar{B} \alpha+\bar{A} \bar{\alpha}
\end{array}\right]=0
$$

Thus, for $a=\alpha, \varphi(s) \equiv \alpha$ is a solution of the initial value problem. Using (3.2) and analytic continuation we obtain $\bar{\alpha}=P \bar{\alpha}+Q \alpha$. Hence $u_{1 z \bar{z}}(0) \bar{\alpha}+u_{1 z z}(0) \alpha=0$. So the vector $[\alpha, \bar{\alpha}]^{T}$ is in the kernel of the Hessian of $u_{1}$ at 0 , and therefore $x_{0}$ is a degenerate critical point of $u_{1}$. The inclusion Ker $H \dot{u}_{0}\left(x_{0}\right) \subseteq \operatorname{Ker} H u_{1}\left(x_{0}\right)$ clearly follows from our arguments.

\section{Proof of Theorem 1.3}

We start out with a Morse function $w: X \rightarrow \mathbb{R}$ with a minimal amount of critical points and distinct critical values. The condition $X \neq \mathbb{C} P^{1}$ implies that $b_{i}(X)>0$ for some $0<i<2 n$. From the Morse inequalities it follows that $v$ has at least one saddle point $x_{0}$. Let $p$ be the index of $w$ at $x_{0}$.

We choose a holomorphic coordinate chart $\left(z_{1}=x_{1}+i y_{1}, \ldots, z_{n}=x_{n}+i y_{n}\right)$ around the point $x_{0}$ such that $\left.\omega_{0}\right|_{x_{0}}=\sum_{j=1}^{n} i d z_{j} \wedge d \bar{z}_{j}$. It is easy to see that one can choose a smooth diffeomorphism $\varphi$ of $X$ such that $\varphi\left(x_{0}\right)=x_{0}$, and in our fixed chart, the Hessian of the Morse function $v=w \circ \varphi$ at $x_{0}$ is diagonal with the following entries on the diagonal:

$\frac{\partial^{2} v}{\partial x_{j}^{2}}\left(x_{0}\right)=\frac{\partial^{2} v}{\partial y_{k}^{2}}\left(x_{0}\right)=1$ if $1 \leq j \leq n, p+1 \leq k \leq n$ and $\frac{\partial^{2} v}{\partial y_{l}^{2}}\left(x_{0}\right)=-1$ if $1 \leq l \leq p$.

By multiplying $v$ with a small constant, we can assume that $v \in \mathcal{H}$. We clearly have $v_{z_{1} \bar{z}_{1}}\left(x_{0}\right)=0$ and $\left|v_{z_{1} z_{1}}\left(x_{0}\right)\right| \neq 0$. We can choose a number $\gamma>0$ such that

$$
\left|v_{z_{1} z_{1}}\left(x_{0}\right)\right|>2 \gamma+v_{z_{1} \bar{z}_{1}}\left(x_{0}\right) \text { and } \gamma+v_{z_{1} \bar{z}_{1}}\left(x_{0}\right)>0 \text {. }
$$

Using this and the fact that $v_{z \bar{z}}\left(x_{0}\right)$ is diagonal, by a lemma that will be provided below, one can find a smooth function $\rho$ supported inside our coordinate patch that is identically equal to 1 in a neighborhood of $x_{0}$ such that

$$
\omega_{0}+i \partial \bar{\partial}(\gamma-1) \rho(z)\left|z_{1}\right|^{2}>0 \text { and }\left(\omega_{0}+i \partial \bar{\partial} v\right)+i \partial \bar{\partial}(\gamma-1) \rho(z)\left|z_{1}\right|^{2}>0 .
$$

Assume that problem (1.1) has a $C^{3}$ solution $u$ with boundary data $u_{0}=$ $(\gamma-1) \rho(z)\left|z_{1}\right|^{2}$ and $u_{1}=v+(\gamma-1) \rho(z)\left|z_{1}\right|^{2}$. By Theorem 1.1, $\dot{u}_{0}$ is a Morse function, and all its critical points are critical points of $v$. As $v$ has the minimal amount of critical points, the critical sets of $\dot{u}_{0}$ and $v$ coincide; in particular, $x_{0}$ is critical for $\dot{u}_{0}$.

Since $\operatorname{rank}(\omega+i \partial \bar{\partial} u) \equiv n$ and $\omega+i \partial \bar{\partial} u$ is semi-positive on the boundary of $\bar{S} \times X$, we obtain that $\omega+i \partial \bar{\partial} u \geq 0$; in particular, $u$ is $\omega$-plurisubharmonic. It follows from this and Lemma 3.1 that all the conditions of Lemma 3.2 in [DL are satisfied for the Kähler metric $\omega^{\prime}=\omega+i \partial \bar{\partial}(\gamma-1) \rho(z)\left|z_{1}\right|^{2}$ and the $\omega^{\prime}$-plurisubharmonic 
function $u^{\prime}=u-(\gamma-1) \rho(z)\left|z_{1}\right|^{2}$. However, the conclusion of this result is violated since for $v^{\prime}=u_{1}^{\prime}$ and $\xi^{\prime}=\partial / \partial z_{1}$ we have

$$
\left|\sum_{j, k} v_{z_{j} z_{k}}^{\prime}\left(x_{0}\right) \xi_{j}^{\prime} \xi_{k}^{\prime}\right|>\sum_{j, k}\left(2 \omega_{j k}^{\prime} \xi_{j}^{\prime}{\overline{\xi^{\prime}}}_{k}+v_{z_{j} \bar{z}_{k}}^{\prime}\left(x_{0}\right) \xi_{j}^{\prime}{\overline{\xi^{\prime}}}_{k}\right) \text {. }
$$

To prove the last statement of the theorem, we refer to elements of Morse theory. If one perturbs slightly $u_{0}$ and $u_{1}$ in the $C^{\infty}$ topology, it is a standard result that $u_{1}-u_{0}$ is still a Morse function with distinct critical values. Also, by GG, Theorem 2.2, p. 79], we know that for small perturbations, $u_{1}-u_{0}$ will still have a minimal amount of critical points. Lastly, if the perturbation is small enough, then $u_{0}, u_{1} \in \mathcal{H}$, and at one of the critical points (4.1) still holds. This completes the main argument of the proof.

As promised, we provide the following lemma initially found by Donaldson [D2, Lemma 8]; see also [LV, Lemma 3.3]. Since its original formulation is slightly inadequate for our purposes, we reformulate it here and give a proof for completeness.

Lemma 4.1. Suppose $\omega^{0}$ and $\omega^{1}$ are two Kähler metrics on a complex manifold $X$. Let $q^{0}$ and $q^{1}$ be two real smooth functions on $X$ with critical points at $x_{0}$ and $q^{0}\left(x_{0}\right)=q^{1}\left(x_{0}\right)=0$. If in some holomorphic coordinate patch $U$ around $x_{0}$ we have $q_{z \bar{z}}^{l}\left(x_{0}\right)(\xi, \bar{\xi})+\omega^{l}\left(x_{0}\right)(\xi, \bar{\xi})>0$ for all nonzero $\xi \in T_{x_{0}} X, l=0,1$, then one can find a smooth function $\rho$ supported in $U$ that is identically 1 in a neighborhood of $x_{0}$ such that $\omega^{l}+i \partial \bar{\partial}\left(\rho q^{l}\right)>0, l=0,1$.

Proof. By shrinking $U$ and possibly rescaling the coordinates, there exists $1>\alpha>0$ such that $\omega^{l}+i \partial \bar{\partial} q^{l}>\alpha \omega^{l}$ for $l=0,1$ on $U$ and the coordinates map $x_{0}$ to 0 and $U$ to a neighborhood of $\{|z| \leq 2\} \subset \mathbb{C}^{n}$.

Now take a smooth function $\beta:[-\infty,+\infty) \rightarrow[0,1]$ such that $\beta(t)=0$ if $t>0$ and $\beta(t)=1$ if $t<-1$. Then $\rho(t)=\beta(\varepsilon \log t), \varepsilon>0$, is supported in $[0,1], \rho(t)=1$ if $t<e^{-1 / \varepsilon}$ and $t\left|\rho^{\prime}(t)\right|, t^{2}\left|\rho^{\prime \prime}(t)\right|<C \varepsilon$ for some constant $C$. From all of this it is easy to see that on $U$ we have

$$
\omega^{l}+i \partial \bar{\partial}\left(\rho\left(|z|^{2}\right) q^{l}\right)>\omega^{l}+i \rho\left(|z|^{2}\right) \partial \bar{\partial} q^{l}-D \varepsilon \omega^{l}>(\alpha-D \varepsilon) \omega^{l}
$$

for some constant $D$ independent of $\varepsilon, l=0,1$. Since $\rho\left(|z|^{2}\right) q^{l}$ is identically 0 outside $U$, for small enough $\varepsilon, \rho\left(|z|^{2}\right)$ has the required properties.

\section{ACKNOWLEDGEMENTS}

The author would like to thank Y. A. Rubinstein for bringing his attention to the paper $\mathrm{RZ}$ and for his patience in conversations related to the topic. As the author found out later, he was developing similar ideas at the time this paper was written. The author would also like to thank L. Lempert for his guidance and for his many useful suggestions.

\section{REFERENCES}

[BK] Eric Bedford and Morris Kalka, Foliations and complex Monge-Ampère equations, Comm. Pure Appl. Math. 30 (1977), no. 5, 543-571. MR0481107 (58 \#1253)

[BT] Eric Bedford and B. A. Taylor, The Dirichlet problem for a complex Monge-Ampère equation, Invent. Math. 37 (1976), no. 1, 1-44. MR0445006 (56 \#3351)

[Ca] Eugenio Calabi, Extremal Kähler metrics, Seminar on Differential Geometry, Ann. of Math. Stud., vol. 102, Princeton Univ. Press, Princeton, N.J., 1982, pp. 259-290. MR645743 (83i:53088) 
[C] Xiuxiong Chen, The space of Kähler metrics, J. Differential Geom. 56 (2000), no. 2, 189234. MR.1863016 (2003b:32031)

[DL] T. Darvas and L. Lempert, Weak geodesics in the space of Kähler metrics, Math. Res. Lett. 19 (2012), no. 5, 1127-1135. MR3039835

[D1] S. K. Donaldson, Symmetric spaces, Kähler geometry and Hamiltonian dynamics, Northern California Symplectic Geometry Seminar, Amer. Math. Soc. Transl. Ser. 2, vol. 196, Amer. Math. Soc., Providence, RI, 1999, pp. 13-33. MR.1736211(2002b:58008)

[D2] S. K. Donaldson, Holomorphic discs and the complex Monge-Ampère equation, J. Symplectic Geom. 1 (2002), no. 2, 171-196. MR.1959581 (2003m:32037)

[F] A. Futaki, An obstruction to the existence of Einstein Kähler metrics, Invent. Math. 73 (1983), no. 3, 437-443, DOI 10.1007/BF01388438. MR718940 (84j:53072)

[GG] M. Golubitsky and V. Guillemin, Stable mappings and their singularities, Graduate Texts in Mathematics, Vol. 14, Springer-Verlag, New York, 1973. MR0341518 (49 \#6269)

$[\mathrm{LV}]$ L. Lempert and L. Vivas, Geodesics in the space of Kähler metrics, Duke Math. J. 162 (2013), no. 7, 1369-1381. MR.3079251

[M] Toshiki Mabuchi, Some symplectic geometry on compact Kähler manifolds. I, Osaka J. Math. 24 (1987), no. 2, 227-252. MR.909015 (88m:53126)

[Ma] Yozô Matsushima, Sur la structure du groupe d'homéomorphismes analytiques d'une certaine variété kählérienne (French), Nagoya Math. J. 11 (1957), 145-150. MR.0094478 (20 \#995)

[RZ] Y. A. Rubinstein and S. Zelditch, The Cauchy problem for the homogeneous Monge-Ampère equation, III. Lifespan, arXiv:1205.4793.

[S] Stephen Semmes, Complex Monge-Ampère and symplectic manifolds, Amer. J. Math. 114 (1992), no. 3, 495-550, DOI 10.2307/2374768. MR1165352(94h:32022)

Department of Mathematics, Purdue University, West Lafayette, Indiana 47907

E-mail address: tdarvas@math.purdue.edu 